\title{
ON THE REALIZABLE CLASSES OF THE SQUARE ROOT OF THE INVERSE DIFFERENT IN THE UNITARY CLASS GROUP
}

\author{
CINDY TSANG
}

\begin{abstract}
Let $K$ be a number field with ring of integers $\mathcal{O}_{K}$ and let $G$ be a finite abelian group of odd order. Given a $G$-Galois $K$-algebra $K_{h}$, let $A_{h}$ denote its square root of the inverse different, which exists by Hilbert's formula. If $K_{h} / K$ is weakly ramified, then the pair $\left(A_{h}, T r_{h}\right)$ is locally $G$-isometric to $\left(\mathcal{O}_{K} G, t_{K}\right)$ and hence defines a class in the unitary class group $\operatorname{UCl}\left(\mathcal{O}_{K} G\right)$ of $\mathcal{O}_{K} G$. Here $T r_{h}$ denotes the trace of $K_{h} / K$ and $t_{K}$ the symmetric bilinear form on $\mathcal{O}_{K} G$ for which $t_{K}(s, t)=\delta_{s t}$ for all $s, t \in G$. We study the collection of all such classes and show that a subset of them is in fact a subgroup of $\operatorname{UCl}\left(\mathcal{O}_{K} G\right)$.
\end{abstract}

\section{Contents}

1. Introduction 2

2. Notation and Conventions 5

3. G-Forms and Unitary Class Groups 6

3.1. G-Forms 6

3.2. Unitary Class Groups 8

4. Galois Algebras and Resolvends 10

5. The Class of the Square Root of the Inverse Different 14

5.1. Computation using Resolvends 14

5.2. Proof of Theorem $1.1 \quad 15$

5.3. Summary of Main Ideas 17

5.4. Cohomology and Reduced Resolvends 18

5.5. The Modified Stickelberger Transpose 21

5.6. Approximation Theorems 24

5.7. Proof of Theorems 1.2 and $1.3 \quad 26$

6. Acknowledgments 28

$\begin{array}{ll}\text { References } & 29\end{array}$

Date: November 29, 2015. 


\section{INTRODUCTION}

Let $K$ be a number field with ring of integers $\mathcal{O}_{K}$ and let $G$ be a finite group of odd order. The set of all isomorphism classes of $G$-Galois $K$-algebras (see Section 4 for a brief review of Galois algebras) is in one-one correspondence with the pointed set $H^{1}\left(\Omega_{K}, G\right)$, where $\Omega_{K}$ is the absolute Galois group of $K$ acting trivially on $G$. Given $h \in H^{1}\left(\Omega_{K}, G\right)$, we will write $K_{h}$ for a Galois algebra representative and $T r_{h}$ for the trace of $K_{h} / K$. Moreover, we will write $A_{h}$ for the square root of the inverse different of $K_{h} / K$. Since $G$ has odd order, the inverse different of $K_{h} / K$ indeed has a square root by Hilbert's formula (see [7, Chapter IV, Proposition 4], for example).

If $K_{h} / K$ is weakly ramified (see Definition 4.4), then it follows from [2, Theorem 1 in Section 2] that $A_{h}$ is locally free as an $\mathcal{O}_{K} G$-module and hence defines a class $\operatorname{cl}\left(A_{h}\right)$ in the locally free class group $\operatorname{Cl}\left(\mathcal{O}_{K} G\right)$ of $\mathcal{O}_{K} G$. Such a class in $\mathrm{Cl}\left(\mathcal{O}_{K} G\right)$ is said to be $A$-realizable, and tame $A$-realizable if $K_{h} / K$ is tame. For $G$ abelian, in [8] the author has studied these $A$-realizable classes using techniques developed by L. McCulloh in [5]. The purpose of this paper is to show that the same methods used in [8] can be applied to study the structure of $A_{h}$ as an $\mathcal{O}_{K} G$-module equipped with the bilinear form induced by $\operatorname{Tr}_{h}$. We will explain this in more detail below.

Given $h \in H^{1}\left(\Omega_{K}, G\right)$, it is well-known that $A_{h}$ is self-dual respect to $\operatorname{Tr}_{h}$ (this follows from $[4$, Chapter 3, (2.14)], for example). In other words, we have

$$
A_{h}=\left\{a \in K_{h} \mid \operatorname{Tr}_{h}\left(a A_{h}\right) \subset \mathcal{O}_{K}\right\} .
$$

In particular, the map $\operatorname{Tr}_{h}$ induces a $G$-invariant symmetric $\mathcal{O}_{K}$-bilinear form

$$
A_{h} \times A_{h} \longrightarrow \mathcal{O}_{K} ; \quad(a, b) \mapsto \operatorname{Tr}_{h}(a b)
$$

on $A_{h}$. The pair $\left(A_{h}, \operatorname{Tr}_{h}\right)$ is thus a $G$-form over $\mathcal{O}_{K}$ (see Subsection 3.1 for a brief review of $G$-forms). On the other hand, there is a canonical symmetric $\mathcal{O}_{K}$-bilinear form $t_{K}$ on $\mathcal{O}_{K} G$ for which $t_{K}(s, t)=\delta_{s t}$ for all $s, t \in G$.

If $K_{h} / K$ is weakly ramified, then again $A_{h}$ is locally free over $\mathcal{O}_{K} G$ by [2, Theorem 1 in Section 2]. In other words, for each prime $v$ in $\mathcal{O}_{K}$, there exists an $\mathcal{O}_{K_{v}} G$-isomorphism $\mathcal{O}_{K_{v}} \otimes_{\mathcal{O}_{K}} A_{h} \simeq \mathcal{O}_{K_{v}} G$, where $\mathcal{O}_{K_{v}}$ denotes the ring 
of integers in the completion of $K$ with respect to $v$. It is natural to ask whether such an isomorphism may be chosen such that the bilinear forms $\operatorname{Tr}_{h}$ and $t_{K}$ are preserved. More precisely, are the $G$-forms $\left(A_{h}, \operatorname{Tr}_{h}\right)$ and $\left(\mathcal{O}_{K} G, t_{K}\right)$ locally $G$-isometric over $\mathcal{O}_{K}$ (see Definition 3.7)? For $G$ abelian and of odd order, the answer turns out to be affirmative (see Subsection 5.1). In this case, the pair $\left(A_{h}, T r_{h}\right)$ defines a class in the unitary class group $U C l\left(\mathcal{O}_{K} G\right)$ of $\mathcal{O}_{K} G$ (see Subsection 3.2). By abuse of terminology, such a class in $\operatorname{UCl}\left(\mathcal{O}_{K} G\right)$ is also said to be $A$-realizable, and tame A-reazliable if $K_{h} / K$ is tame.

In what follows, we assume that $G$ is abelian and of odd order. The pointed set $H^{1}\left(\Omega_{K}, G\right)$ is then equal to $\operatorname{Hom}\left(\Omega_{K}, G\right)$ and hence has a group structure. Define

$$
H_{t}^{1}\left(\Omega_{K}, G\right):=\left\{h \in H^{1}\left(\Omega_{K}, G\right) \mid K_{h} / K \text { is tame }\right\},
$$

which is a subgroup of $H^{1}\left(\Omega_{K}, G\right)$ (see Remark 4.5), and

$$
H_{w}^{1}\left(\Omega_{K}, G\right):=\left\{h \in H^{1}\left(\Omega_{K}, G\right) \mid K_{h} / K \text { is weakly ramified }\right\} .
$$

Moreover, we will write

$$
\mathcal{A}_{u}\left(\mathcal{O}_{K} G\right):=\left\{\operatorname{ucl}\left(A_{h}\right): h \in H_{w}^{1}\left(\Omega_{K}, G\right)\right\}
$$

for the set of all $A$-realizable classes in $\operatorname{UCl}\left(\mathcal{O}_{K} G\right)$, and

$$
\mathcal{A}_{u}^{t}\left(\mathcal{O}_{K} G\right):=\left\{\operatorname{ucl}\left(A_{h}\right): h \in H_{t}^{1}\left(\Omega_{K}, G\right)\right\}
$$

for the subset of $\mathcal{A}_{u}\left(\mathcal{O}_{K} G\right)$ consisting of the tame $A$-realizable classes. It has been shown in $\left[3\right.$, Theorem 4.1] that $\mathcal{A}_{u}(\mathbb{Z} G)=1$, and in [6, Theorem 3.6] that $\mathcal{A}_{u}^{t}\left(\mathcal{O}_{K} G\right)$ is subgroup of $\operatorname{UCl}\left(\mathcal{O}_{K} G\right)$ when $G$ has odd prime order and $K$ contains all $|G|$-th roots of unity.

Now, consider the map

$$
\operatorname{gal}_{A, u}: H_{w}^{1}\left(\Omega_{K}, G\right) \longrightarrow \operatorname{UCl}\left(\mathcal{O}_{K} G\right) ; \quad \operatorname{gal}_{A, u}(h):=\operatorname{ucl}\left(A_{h}\right) .
$$

Note that gal ${ }_{A, u}\left(H_{w}^{1}\left(\Omega_{K}, G\right)\right)=\mathcal{A}_{u}\left(\mathcal{O}_{K} G\right)$ and gal ${ }_{A, u}\left(H_{t}^{1}\left(\Omega_{K}, G\right)\right)=\mathcal{A}_{u}^{t}\left(\mathcal{O}_{K} G\right)$ by definition. But $H_{w}^{1}\left(\Omega_{K}, G\right)$ is only a subset of $H^{1}\left(\Omega_{K}, G\right)$, and the map gal $_{A, u}$ is not a homomorphism in general even when restricted to the subgroup $H_{t}^{1}\left(\Omega_{K}, G\right)$. Hence, it is unclear whether $\mathcal{A}_{u}\left(\mathcal{O}_{K} G\right)$ and $\mathcal{A}_{u}^{t}\left(\mathcal{O}_{K} G\right)$ are 
subgroups of $\operatorname{UCl}\left(\mathcal{O}_{K} G\right)$. Nevertheless, we will show that $\operatorname{gal}_{A, u}$ preserves inverses and is weakly multiplicative. More precisely, let $M_{K}$ be the set of primes in $\mathcal{O}_{K}$ and define

$$
d(h):=\left\{v \in M_{K} \mid K_{h} / K \text { is ramified at } v\right\}
$$

for $h \in H^{1}\left(\Omega_{K}, G\right)$. Analogous to [8, Theorem 1.2], we will prove:

Theorem 1.1. Let $K$ be a number field and let $G$ be a finite abelian group of odd order. For all $h, h_{1}, h_{2} \in H_{w}^{1}\left(\Omega_{K}, G\right)$ with $d\left(h_{1}\right) \cap d\left(h_{2}\right)=\emptyset$, we have

(a) $h^{-1} \in H_{w}^{1}\left(\Omega_{K}, G\right)$ and $\operatorname{gal}_{A, u}\left(h^{-1}\right)=\operatorname{gal}_{A, u}(h)^{-1}$;

(b) $h_{1} h_{2} \in H_{w}^{1}\left(\Omega_{K}, G\right)$ and $\operatorname{gal}_{A, u}\left(h_{1} h_{2}\right)=\operatorname{gal}_{A, u}\left(h_{1}\right) g a l_{A, u}\left(h_{2}\right)$.

Analogous to [8, Theorems 1.3 and 1.6], we will also give a characterization of the set $\mathcal{A}_{u}^{t}\left(\mathcal{O}_{K} G\right)$ (see $\left.(5.17)\right)$ and then prove:

Theorem 1.2. Let $K$ be a number field and let $G$ be a finite abelian group of odd order. Then, the set $\mathcal{A}_{u}^{t}\left(\mathcal{O}_{K} G\right)$ is a subgroup of $U C l\left(\mathcal{O}_{K} G\right)$. Moreover, given $c \in \mathcal{A}_{u}^{t}\left(\mathcal{O}_{K} G\right)$ and a finite set $T$ of primes in $\mathcal{O}_{K}$, there exists $h \in$ $H_{t}^{1}\left(\Omega_{K}, G\right)$ such that

(1) $K_{h} / K$ is a field extension;

(2) $K_{h} / K$ is unramified at all $v \in T$;

(3) $c=u c l\left(A_{h}\right)$.

Theorem 1.3. Let $K$ be a number field and let $G$ be a finite abelian group of odd order. Let $h \in H_{w}^{1}\left(\Omega_{K}, G\right)$ and let $V$ denote the set of primes in $\mathcal{O}_{K}$ which are wildly ramified in $K_{h} / K$. If

(1) every $v \in V$ is unramified over $\mathbb{Q}$; and

(2) the ramification index of every $v \in V$ in $K_{h} / K$ is prime,

then we have $\operatorname{ucl}\left(A_{h}\right) \in \mathcal{A}_{u}^{t}\left(\mathcal{O}_{K} G\right)$.

Remark 1.4. Notice that there is a natural homomorphism

$$
\Phi: \mathrm{UCl}\left(\mathcal{O}_{K} G\right) \longrightarrow \mathrm{Cl}\left(\mathcal{O}_{K} G\right) ; \quad \operatorname{ucl}((X, T)) \mapsto \operatorname{cl}(X)
$$

afforded by forgetting the given $G$-invariant symmetric $\mathcal{O}_{K}$-bilinear form $T$ on $X$ for any locally free $\mathcal{O}_{K} G$-module $X$. Theorems $1.1,1.2$, and 1.3 are 
therefore refinements of [8, Theorems 1.2, 1.3, and 1.6], respectively. In fact, their proofs are essentially the same.

Here is a brief outline of the contents of the rest of this paper. In Section 3, we will define the unitary class group, which was first introduced by J. Morales in [6]. In Section 4, we will give a brief review of Galois algebras and resolvends. Then, in Section 5, we will recall the necessary definitions to give a characterization of the set $\mathcal{A}_{u}^{t}\left(\mathcal{O}_{K} G\right)$ (see (5.17)). We will prove Theorem 1.1 in Subsection 5.2, and then Theorems 1.2 and 1.3 in Subsection 5.7. To avoid repetition, we will use certain results from [8] without restating them.

\section{Notation and Conventions}

Throughout this paper, we will fix a number field $K$ and a finite group $G$. We will also use the convention that the homomorphisms in the cohomology groups considered are all continuous.

The symbol $F$ will denote a number field or a finite extension of $\mathbb{Q}_{p}$, where $p$ is a prime number. Given such an $F$, we will define:

$\mathcal{O}_{F}:=$ the ring of integers in $F$;

$F^{c}:=$ a fixed algebraic closure of $F$;

$\Omega_{F}:=\operatorname{Gal}\left(F^{c} / F\right)$;

$F^{t}:=$ the maximal tamely ramified extension of $F$ in $F^{c}$;

$\Omega_{F}^{t}:=\operatorname{Gal}\left(K^{t} / K\right)$;

$M_{F}:=$ the set of all finite primes in $F$;

$[-1]:=$ the involution on $F^{c} G$ induced by the involution $s \mapsto s^{-1}$ on $G$;

$t_{F}:=$ the symmetric $\mathcal{O}_{F}$-bilinear form $\mathcal{O}_{F} G \times \mathcal{O}_{F} G \longrightarrow \mathcal{O}_{F}$ for which $t_{F}(s, t)=\delta_{s t}$ for all $s, t \in G$.

For $G$ abelian, we will further define:

$\widehat{G}:=$ the group of irreducible $F^{c}$-valued characters on $G$. 
We will also let $\Omega_{F}$ and $\Omega_{F}^{t}$ act trivially on $G$ from the left. Moreover, we will choose a compatible set $\left\{\zeta_{n}: n \in \mathbb{Z}^{+}\right\}$of primitive roots of unity in $F^{c}$.

For $F$ a number field and $v \in M_{F}$, we adopt the following notation:

$$
\begin{aligned}
& F_{v}:= \text { the completion of } F \text { with respect to } v ; \\
& i_{v}:=\text { a fixed embedding } F^{c} \longrightarrow F_{v}^{c} \text { extending the natural } \text { embedding } F \longrightarrow F_{v} ; \\
& \widetilde{i_{v}}:=\text { the embedding } \Omega_{F_{v}} \longrightarrow \Omega_{F} \text { induced by } i_{v} .
\end{aligned}
$$

Moreover, if $\left\{\zeta_{n}: n \in \mathbb{Z}^{+}\right\}$is the chosen compatible set of primitive roots of unity in $F^{c}$, then for each $v \in M_{F}$ we choose $\left\{i_{v}\left(\zeta_{n}\right): n \in \mathbb{Z}^{+}\right\}$to be the compatible set of primitive roots of unity in $F_{v}^{c}$.

\section{G-Forms and Unitary Class Groups}

3.1. G-Forms. Let $F$ be a number field or a finite extension of $\mathbb{Q}_{p}$. We will give a brief review of $G$-forms over $\mathcal{O}_{F}$ and their basic properties.

Definition 3.1. An $\mathcal{O}_{F} G$-lattice is a left $\mathcal{O}_{F} G$-module which is finitely generated and projective as an $\mathcal{O}_{F}$-module.

Definition 3.2. A $G$-form over $\mathcal{O}_{F}$ is pair $(X, T)$, where $X$ is an $\mathcal{O}_{F} G$-lattice and $T: X \times X \longrightarrow \mathcal{O}_{F}$ is $G$-invariant symmetric $\mathcal{O}_{F}$-bilinear form on $X$. Two $G$-forms $(X, T)$ and $\left(X^{\prime}, T^{\prime}\right)$ over $\mathcal{O}_{F}$ are said to be $G$-isometric over $\mathcal{O}_{F}$ if there exists an isomorphism $\varphi: X \longrightarrow X^{\prime}$ of $\mathcal{O}_{F} G$-modules such that

$$
T^{\prime}(\varphi(x), \varphi(y))=T(x, y) \quad \text { for all } x, y \in X \text {. }
$$

Such an isomorphism is called a $G$-isometry over $\mathcal{O}_{F}$. The $G$-isometry class of $(X, T)$ will be denoted by $\operatorname{ucl}((X, T))$.

Given a $G$-form $(X, T)$ over $\mathcal{O}_{F}$, notice that the map $T$ extends uniquely to a $G$-invariant symmetric $F$-bilinear on $F \otimes_{\mathcal{O}_{F}} X$ via linearity. By abuse of notation, we will still use $T$ to denote this bilinear form. 
Definition 3.3. Let $(X, T)$ be a $G$-form over $\mathcal{O}_{F}$. The dual of $X$ with respect to $T$, or simply the dual of $X$, is defined to be the $\mathcal{O}_{F}$-module

$$
X^{*}:=\left\{x \in F \otimes_{\mathcal{O}_{F}} X \mid T(x, X) \subset \mathcal{O}_{F}\right\} .
$$

The $G$-form $(X, T)$ is said to be self-dual with respect to $T$, or simply self-dual if $X=X^{*}$. Moreover, an element $x \in F \otimes_{\mathcal{O}_{F}} X$ is said to be self dual with respect to $T$, or simply self-dual if

$$
T(s x, t x)=\delta_{s t} \quad \text { for all } s, t \in G .
$$

Recall that $t_{F}$ denotes the canonical symmetric $\mathcal{O}_{F}$-bilinear form on $\mathcal{O}_{F} G$ for which $t_{F}(s, t)=\delta_{s t}$ for all $s, t \in G$. The $G$-forms $(X, T)$ over $\mathcal{O}_{F}$ which are $G$-isometric to $\left(\mathcal{O}_{F} G, t_{F}\right)$ are precisely those for which $X$ has a free self-dual generator over $\mathcal{O}_{F} G$.

Proposition 3.4. A $G$-form $(X, T)$ over $\mathcal{O}_{F}$ is $G$-isometric to $\left(\mathcal{O}_{F} G, t_{F}\right)$ if and only if there exists $x \in X$ such that $X=\mathcal{O}_{F} G \cdot x$ and $x$ is self-dual.

Proof. If $(X, T)$ is $G$-isometric to $\left(\mathcal{O}_{F} G, t_{F}\right)$ and $\varphi: \mathcal{O}_{F} G \longrightarrow X$ is a $G$ isometry over $\mathcal{O}_{F}$, then clearly $x:=\varphi(1)$ is self-dual and $X=\mathcal{O}_{F} G \cdot x$. Conversely, if $x \in X$ is self-dual and $X=\mathcal{O}_{F} G \cdot x$, then

$$
\varphi: \mathcal{O}_{F} G \longrightarrow X ; \quad \varphi(\gamma):=\gamma \cdot x
$$

is a $G$-isometry over $\mathcal{O}_{F}$. This proves the claim.

Recall that $[-1]$ denotes the involution on $F^{c} G$ induced by the involution $s \mapsto s^{-1}$ on $G$. This involution may be used to identify elements $c \in(F G)^{\times}$ for which $\mathcal{O}_{F} G \cdot c$ is self-dual and those which are self-dual.

Proposition 3.5. Let $c \in(F G)^{\times}$.

(a) The $\mathcal{O}_{F} G$-lattice $\mathcal{O}_{F} G \cdot c$ is self-dual if and only if $c c^{[-1]} \in\left(\mathcal{O}_{F} G\right)^{\times}$.

(b) The element $c$ is self-dual if and only if $c c^{[-1]}=1$.

Proof. An element $\gamma \in F G$ lies in $\gamma \in\left(\mathcal{O}_{F} G \cdot c\right)^{*}$ if and only if

$$
t_{F}(\gamma, s c) \in \mathcal{O}_{F} \quad \text { for all } s \in G
$$


because $t_{F}$ is $\mathcal{O}_{F}$-bilinear. But for any $s \in G$, we have $t_{F}(\gamma, s c)=t_{F}\left(\gamma c^{[-1]}, s\right)$, which is simply the coefficient of $s$ in $\gamma c^{[-1]}$. Hence, condition (3.1) holds precisely when $\gamma c^{[-1]} \in \mathcal{O}_{F} G$. This means that

$$
\left(\mathcal{O}_{F} G \cdot c\right)^{*}=\mathcal{O}_{F} G \cdot\left(c^{[-1]}\right)^{-1}
$$

and so $\mathcal{O}_{F} G \cdot c$ is self-dual if and only if $c c^{[-1]} \in\left(\mathcal{O}_{F} G\right)^{\times}$. This proves (a).

For (b), simply observe that

$$
t_{F}(s c, t c)=t_{F}\left(s \cdot c c^{[-1]}, t\right) \quad \text { for all } s, t \in G .
$$

It follows that $c$ is self-dual if and only if $c c^{[-1]}=1$.

Definition 3.6. In view of Proposition 3.5, define

$$
\begin{aligned}
& F G_{(s)}:=\left\{c \in(F G)^{\times} \mid c c^{[-1]} \in\left(\mathcal{O}_{F} G\right)^{\times}\right\} ; \\
& F G_{(1)}:=\left\{c \in(F G)^{\times} \mid c c^{[-1]}=1\right\} .
\end{aligned}
$$

Now, assume that $F$ is a number field. Given a $G$-form $(X, T)$ over $\mathcal{O}_{F}$, notice that the map $T$ extends uniquely to a $G$-invariant symmetric $\mathcal{O}_{F_{v}}{ }^{-}$ bilinear on $X_{v}:=\mathcal{O}_{F_{v}} \otimes_{\mathcal{O}_{F}} X$ via linearity for each $v \in M_{F}$. We will denote these bilinear forms by $T_{v}$.

Definition 3.7. A $G$-form $(X, T)$ over $\mathcal{O}_{F}$ is said to be locally $G$-isometric to $\left(\mathcal{O}_{F} G, t_{F}\right)$ if $\left(X_{v}, T_{v}\right)$ and $\left(\mathcal{O}_{F_{v}} G, t_{F_{v}}\right)$ are $G$-isometric over $\mathcal{O}_{F_{v}}$ for all $v \in M_{F}$. We will write $g\left(\mathcal{O}_{F} G\right)_{s}$ for the set of all such $G$-forms $(X, T)$ over $\mathcal{O}_{F}$ that is $G$-isometric to $\left(\mathcal{O}_{F} G \cdot c, t_{F}\right)$ for some $c \in J(F G)$.

3.2. Unitary Class Groups. Let $F$ be a number field. Moreover, assume that $G$ is abelian and of odd order. Following [6, Section], we will define the unitary class group of $\mathcal{O}_{F} G$. Our approach will be slightly different, but the resulting group is canonically isomorphic to that defined in [6].

As a set, the unitary class group of $\mathcal{O}_{F} G$ is defined to be

$$
\mathrm{UCl}\left(\mathcal{O}_{F} G\right):=\left\{\operatorname{ucl}((X, T)):(X, T) \in g\left(\mathcal{O}_{F} G\right)_{s}\right\}
$$

We will show that this set has a group structure by giving it an idèlic description. The key lies in the following theorem. 
Theorem 3.8. Let $X$ be an $\mathcal{O}_{F} G$-lattice in $F G$. We have $\left(X, t_{F}\right) \in g\left(\mathcal{O}_{F} G\right)_{s}$ if and only if $X$ is locally free over $\mathcal{O}_{F} G$ and self-dual with respect to $t_{F}$.

Proof. If $\left(X, t_{F}\right) \in g\left(\mathcal{O}_{F} G\right)_{s}$, then $X$ is locally free over $\mathcal{O}_{F} G$ and self-dual with respect to $t_{F}$ by Proposition 3.4. As for the converse, see [3, Corollary 2.4]; we remark that this requires that $G$ is abelian and of odd order.

Next, we give an idèlic description of the set $\operatorname{UCl}\left(\mathcal{O}_{K} G\right)$.

Definition 3.9. Let $J(F G)$ and $J\left(F G_{(s)}\right)$ be the restricted direct products of the groups $\left(F_{v} G\right)^{\times}$and $F_{v} G_{(s)}$, respectively, with respect to the subgroups $\left(\mathcal{O}_{F_{v}} G\right)^{\times}$for $v \in M_{F}$. Moreover, let

$$
\partial:(F G)^{\times} \longrightarrow J(F G)
$$

be the diagonal map and let

$$
U\left(\mathcal{O}_{F} G\right):=\prod_{v \in M_{F}}\left(\mathcal{O}_{F_{v}} G\right)^{\times}
$$

be the group of unit idèles.

For each idèle $c \in J(F G)$, define

$$
\mathcal{O}_{F} G \cdot c:=\left(\bigcap_{v \in M_{F}} \mathcal{O}_{F_{v}} G \cdot c_{v}\right) \cap F G .
$$

It is well-known that the locally free $\mathcal{O}_{F} G$-lattices in $F G$ are all of this form.

Proposition 3.10. Let $c, c^{\prime} \in J(F G)$.

(a) The $G$-form $\left(\mathcal{O}_{F} G \cdot c, t_{F}\right)$ belongs to $g\left(\mathcal{O}_{F} G\right)_{s}$ if and only if $c \in J\left(F G_{(s)}\right)$.

(b) The $G$-forms $\left(\mathcal{O}_{F} G \cdot c, t_{F}\right)$ and $\left(\mathcal{O}_{F} G \cdot c^{\prime}, t_{F}\right)$ are $G$-isometric over $\mathcal{O}_{F}$ if and only if

$$
c^{\prime} c^{-1} \in \partial\left(F G_{(1)}\right) U\left(\mathcal{O}_{F} G\right)
$$

Proof. For (a), it follows directly from Proposition 3.5 (a) and Theorem 3.8. As for (b), observe that an isomorphism $\mathcal{O}_{F} G \cdot c \longrightarrow \mathcal{O}_{F} G \cdot c^{\prime}$ is of the form

$$
\varphi: \mathcal{O}_{F} G \cdot c \longrightarrow \mathcal{O}_{F} G \cdot c^{\prime} ; \quad \varphi(x):=\gamma \cdot x
$$


where $\gamma \in(F G)^{\times}$. Moreover, the map $\varphi$ is well-defined and is an isomorphism of $\mathcal{O}_{F} G$-modules if and only if

$$
c^{\prime} c^{-1} \in \partial(\gamma) \cdot U\left(\mathcal{O}_{F} G\right)
$$

Morever, since $t_{F}$ is $\mathcal{O}_{F}$-bilinear and $G$-invariant, the map $\varphi$ is a $G$-isometry over $\mathcal{O}_{F}$ if and only if

$$
t_{F}(\gamma \cdot c, \gamma \cdot s c)=t_{F}(c, s c) \quad \text { for all } s \in G .
$$

But the above simplifies to

$$
t_{F}\left(\gamma \gamma^{[-1]} c c^{[-1]}, s\right)=t_{F}\left(c c^{[-1]}, s\right) \quad \text { for all } s \in G,
$$

which holds precisely when $\gamma \gamma^{[-1]} c c^{[-1]}=c c^{[-1]}$, or equivalently $\gamma \gamma^{[-1]}=1$. This shows that $\varphi$ is a $G$-isometry over $\mathcal{O}_{F}$ if and only if $\gamma \in F G_{(1)}$, and the claim now follows from (3.2).

Now, consider the map

$$
j_{(s)}: J\left(F G_{(s)}\right) \longrightarrow \mathrm{UCl}\left(\mathcal{O}_{F} G\right) ; \quad j_{(s)}(c):=\operatorname{ucl}\left(\left(\mathcal{O}_{F} G \cdot c, t_{F}\right)\right) .
$$

By Proposition 3.10, the map $j_{(s)}$ is well-defined and it induces an injection

$$
\frac{J\left(F G_{(s)}\right)}{\partial\left(F G_{(1)}\right) U\left(\mathcal{O}_{F} G\right)} \longrightarrow \operatorname{UCl}\left(\mathcal{O}_{F} G\right) \text {. }
$$

By definition of $g\left(\mathcal{O}_{F} G\right)_{s}$, the above is also a surjection and hence a bijection. Since the quotient on the left is a group, this bijection induces a group structure on $\operatorname{UCl}\left(\mathcal{O}_{F} G\right)$.

Definition 3.11. The unitary class group of $\mathcal{O}_{F} G$ is defined to be the set

$$
\mathrm{UCl}\left(\mathcal{O}_{F} G\right):=\left\{\operatorname{ucl}((X, T)):(X, T) \in g\left(\mathcal{O}_{F} G\right)_{s}\right\}
$$

equipped with the group structure induced by the bijection (3.3).

\section{Galois Algebras and Resolvends}

Let $F$ be a number field or a finite extension of $\mathbb{Q}_{p}$. The group $G$ is arbitrary for the moment, but will be soon assumed to be abelian. We will give a brief review of Galois algebras and resolvends (see [5, Section 1] for more details). 
Definition 4.1. A Galois algebra over $F$ with group $G$ or $G$-Galois F-algebra is a commutative semi-simple $F$-algebra $N$ on which $G$ acts on the left as a group of automorphisms such that $N^{G}=F$ and $[N: F]=|G|$. Two G-Galois $F$-algebras are isomorphic if there is an $F$-algebra isomorphism between them which preserves the action of $G$.

Recall that $\Omega_{F}$ acts trivially on $G$. Then, the set of isomorphism classes of $G$-Galois $F$-algebras is in one-one correspondence with the pointed set

$$
H^{1}\left(\Omega_{F}, G\right):=\operatorname{Hom}\left(\Omega_{F}, G\right) / \operatorname{Inn}(G) .
$$

In particular, each $h \in \operatorname{Hom}\left(\Omega_{F}, G\right)$ is associated to the $F$-algebra

$$
F_{h}:=\operatorname{Map}_{\Omega_{F}}\left({ }^{h} G, F^{c}\right),
$$

where ${ }^{h} G$ is the group $G$ endowed with the $\Omega_{F}$-action given by

$$
(\omega \cdot s):=h(\omega) s \quad \text { for } s \in G \text { and } \omega \in \Omega_{F} .
$$

The $G$-action on $F_{h}$ is defined by

$$
(s \cdot a)(t):=a(t s) \quad \text { for } a \in F_{h} \text { and } s, t \in G \text {. }
$$

Now, choose a set $\left\{s_{i}\right\}$ of coset representatives for $h\left(\Omega_{F}\right) \backslash G$, then each $a \in F$ is determined by the values $a\left(s_{i}\right)$, and clearly each $a\left(s_{i}\right)$ may be arbitrarily chosen provided that it is fixed by all $\omega \in \operatorname{ker}(h)$. Hence, if

$$
F^{h}:=\left(F^{c}\right)^{\operatorname{ker}(h)},
$$

then the choices of the coset representatives $\left\{s_{i}\right\}$ induce an isomorphism

$$
F_{h} \simeq \prod_{h\left(\Omega_{F}\right) \backslash G} F^{h}
$$

of $F$-algebras. Since $h$ induces an isomorphism $\operatorname{Gal}\left(F^{h} / F\right) \simeq h\left(\Omega_{F}\right)$, we have

$$
\left[F_{h}: F\right]=\left[G: h\left(\Omega_{F}\right)\right]\left[F^{h}: F\right]=|G| .
$$

Viewing $F$ as embedded in $F_{h}$ as the constant $F$-valued functions, we have $F_{h}^{G}=F$ as well. Hence, indeed $F_{h}$ is a $G$-Galois $F$-algebra. 
It is not difficult to verify that every $G$-Galois $F$-algebra is isomorphic to some $F_{h}$ arising from a homomorphism $h \in \operatorname{Hom}\left(\Omega_{F}, G\right)$, and that for $h, h^{\prime} \in \operatorname{Hom}\left(\Omega_{F}, G\right)$ we have $F_{h} \simeq F_{h^{\prime}}$ if and only if $h$ and $h^{\prime}$ differ by an element in $\operatorname{Inn}(G)$. Hence, indeed the set of isomorphism classes of $G$-Galois $F$-algebras is in bijection with (4.1).

In the rest of this section, we will assume that $G$ is abelian. In this case, the pointed set $H^{1}\left(\Omega_{F}, G\right)$ is equal to $\operatorname{Hom}\left(\Omega_{F}, G\right)$ and in particular is a group.

Definition 4.2. Given $h \in \operatorname{Hom}\left(\Omega_{F}, G\right)$, let $F^{h}$ be as in (4.2). Let $\mathcal{O}^{h}:=\mathcal{O}_{F^{h}}$ and define the ring of integers of $F_{h}$ by

$$
\mathcal{O}_{h}:=\operatorname{Map}_{\Omega_{F}}\left({ }^{h} G, \mathcal{O}^{h}\right) .
$$

If the inverse different of $F^{h} / F$ has a square root, denote it by $A^{h}$ and define the square root of the inverse different of $F_{h} / F$ by

$$
A_{h}:=\operatorname{Map}_{\Omega_{F}}\left({ }^{h} G, A^{h}\right) .
$$

Remark 4.3. For $F$ a number field and $h \in \operatorname{Hom}\left(\Omega_{F}, G\right)$, define

$$
h_{v} \in \operatorname{Hom}\left(\Omega_{F_{v}}, G\right) ; \quad h_{v}:=h \circ \widetilde{i_{v}}
$$

for each $v \in M_{F}$. It is proved in $[5,(1.4)]$ that

$$
\left(F_{v}\right)_{h_{v}} \simeq F_{v} \otimes_{F} F_{h}
$$

Consequently, we have

$$
\begin{aligned}
\mathcal{O}_{h_{v}} & \simeq \mathcal{O}_{F_{v}} \otimes_{\mathcal{O}_{F}} \mathcal{O}_{h} \\
A_{h_{v}} & \simeq \mathcal{O}_{F_{v}} \otimes_{\mathcal{O}_{F}} A_{h}
\end{aligned}
$$

where we implicitly assume that $A^{h}$ and $A^{h_{v}}$ exist in the second isomorphism.

Definition 4.4. Given $h \in \operatorname{Hom}\left(\Omega_{F}, G\right)$, we say that $F_{h} / F$ or $h$ is unramified if $F^{h} / F$ is unramified. Similarly for tame, wild, and weakly ramified. Recall that a Galois extension over $F$ is said to be weakly ramified if all of the second ramification groups (in lower numbering) attached to it are trivial.

Remark 4.5. A homomorphism $h \in \operatorname{Hom}\left(\Omega_{F}, G\right)$ is tame if and only if it factors through the quotient map $\Omega_{F} \longrightarrow \Omega_{F}^{t}$. Hence, the subset of $\operatorname{Hom}\left(\Omega_{F}, G\right)$ 
consisting of the tame homomorphisms may be naturally identified with $\operatorname{Hom}\left(\Omega_{F}^{t}, G\right)$, and is in particular a subgroup of $\operatorname{Hom}\left(\Omega_{F}, G\right)$.

Next, consider the $F^{c}$-algebra $\operatorname{Map}\left(G, F^{c}\right)$ on which we let $G$ act via

$$
(s \cdot a)(t):=a(t s) \quad \text { for } a \in \operatorname{Map}\left(\Omega_{F}, G\right) \text { and } s, t \in G \text {. }
$$

Note that $F_{h}$ is an $F G$-submodule of $\operatorname{Map}\left(G, F^{c}\right)$ for all $h \in \operatorname{Hom}\left(\Omega_{F}, G\right)$.

Definition 4.6. The resolvend map $\mathbf{r}_{G}: \operatorname{Map}\left(G, F^{c}\right) \longrightarrow F^{c} G$ is defined by

$$
\mathbf{r}_{G}(a):=\sum_{s \in G} a(s) s^{-1} .
$$

It is clear that $\mathbf{r}_{G}$ is an isomorphism of $F^{c} G$-modules, but not an isomorphism of $F^{c} G$-algebras because it does not preserve multiplication. Moreover, given $a \in \operatorname{Map}\left(G, F^{c}\right)$, we have that $a \in F_{h}$ if and only if

$$
\omega \cdot \mathbf{r}_{G}(a)=\mathbf{r}_{G}(a) h(\omega) \quad \text { for all } \omega \in \Omega_{F} .
$$

In particular, if $\mathbf{r}_{G}(a)$ is invertible, then $h$ is given by

$$
h(\omega)=\mathbf{r}_{G}(a)^{-1}\left(\omega \cdot \mathbf{r}_{G}(a)\right) \quad \text { for all } \omega \in \Omega_{F} .
$$

The next proposition shows that resolvends may be used to identify elements $a \in F_{h}$ for which $F_{h}=F G \cdot a$ or $\mathcal{O}_{h}=\mathcal{O}_{F} G \cdot a$.

Proposition 4.7. Let $a \in F_{h}$.

(a) We have $F_{h}=F G \cdot a$ if and only if $\mathbf{r}_{G}(a) \in\left(F^{c} G\right)^{\times}$.

(b) We have $\mathcal{O}_{h}=\mathcal{O}_{F} G \cdot$ a with $h$ unramified if and only if $\mathbf{r}_{G}(a) \in\left(\mathcal{O}_{F^{c}} G\right)^{\times}$.

Furthermore, if $F$ is a finite extension of $\mathbb{Q}_{p}$ and $h$ is unramified, then there exists $a \in \mathcal{O}_{h}$ such that $\mathcal{O}_{h}=\mathcal{O}_{F} G \cdot a$.

Proof. See [5, Proposition 1.8] for (a) and [5, (2.11)] for the first claim in (b). As for the second claim in (b), it follows from a classical theorem of Noether, or alternatively from [5, Proposition 5.5].

Now, let $\operatorname{Tr}: \operatorname{Map}\left(G, F^{c}\right) \longrightarrow F^{c} G$ be the standard algebra trace map defined by

$$
\operatorname{Tr}(a):=\sum_{s \in G} a(s)
$$


This restricts to the trace $T r_{h}: F_{h} \longrightarrow F$ of $F_{h}$ for each $h \in \operatorname{Hom}\left(\Omega_{F}, G\right)$. By abuse of notation, we will also write $T r_{h}$ for the $G$-invariant symmetric $F$-bilinear form $(a, b) \mapsto \operatorname{Tr}_{h}(a b)$ on $F_{h}$ induced by $\operatorname{Tr}_{h}$.

Resolvends may also be used to identify elements $a \in F_{h}$ for which $\mathcal{O}_{F} G \cdot a$ is a full self-dual $\mathcal{O}_{F} G$-lattice in $F_{h}$ and those which are self-dual with respect to $\operatorname{Tr}_{h}$ (cf. Proposition 3.5). Notice that $\mathcal{O}_{F} G \cdot a$ is a full $\mathcal{O}_{F} G$-lattice in $F_{h}$ if and only if $\mathbf{r}_{G}(a) \in\left(F^{c} G\right)^{\times}$by Proposition 4.7 (a).

Proposition 4.8. Let $a \in F_{h}$ be such that $\mathbf{r}_{G}(a) \in\left(F^{c} G\right)^{\times}$.

(a) The $\mathcal{O}_{F} G$-lattice $\mathcal{O}_{F} G \cdot a$ is self-dual if and only if $\mathbf{r}_{G}(a) \mathbf{r}_{G}(a)^{[-1]} \in$ $\left(\mathcal{O}_{F} G\right)^{\times}$.

(b) The element $a$ is self-dual if and only if $\mathbf{r}_{G}(a) \mathbf{r}_{G}(a)^{[-1]}=1$.

Proof. See [8, Proposition 2.8] for (a). As for (b), it follows directly from the simple calculation that

$$
\mathbf{r}_{G}(a) \mathbf{r}_{G}(b)^{[-1]}=\sum_{s \in G} \operatorname{Tr}((s \cdot a) b) s^{-1} \in F G
$$

for all $a, b \in F_{h}$.

\section{The Class of the Square Root of the Inverse Different}

5.1. Computation using Resolvends. Let $F$ be a number field. Moreover, assume that $G$ is abelian and of odd order. Below, we explain why $\left(A_{h}, \operatorname{Tr}_{h}\right)$ is locally $G$-isometric to $\left(\mathcal{O}_{F} G, t_{F}\right)$ for $h \in \operatorname{Hom}\left(\Omega_{F}, G\right)$ weakly ramified and how the class $\operatorname{ucl}\left(A_{h}\right)$ it defines in $\operatorname{UCl}\left(\mathcal{O}_{F} G\right)$ may be computed using resolvends.

Let $h \in \operatorname{Hom}\left(\Omega_{F}, G\right)$ be weakly ramified. Recall that $A_{h}$ is locally free over $\mathcal{O}_{F} G$ by $\left[2\right.$, Theorem 1 in Section 2] in this case, and that $\mathcal{O}_{F_{v}} \otimes_{\mathcal{O}_{F}} A_{h} \simeq A_{h_{v}}$ from Remark 4.3. Hence, for each $v \in M_{F}$, there exists $a_{v} \in A_{h_{v}}$ such that

$$
A_{h_{v}}=\mathcal{O}_{F_{v}} G \cdot a_{v}
$$

Moreover, by the Normal Basis Theorem, there exists $b \in F_{h}$ such that

$$
F_{h}=F G \cdot b .
$$


Since $G$ has odd order, it follows from [1, Proposition 5.1] that $b \in F_{h}$ may be chosen to be self-dual. Notice that $F_{v} G \cdot a_{v}=F_{h_{v}}=F_{v} G \cdot b$ for all $v \in M_{F}$ and that $\mathcal{O}_{F_{v}} G \cdot a_{v}=\mathcal{O}_{F_{v}} G \cdot b$ for all but finitely may $v \in M_{F}$. This implies that there exists $c \in J(F G)$ such that

$$
a_{v}=c_{v} \cdot b
$$

for $v \in M_{F}$. In particular, the isomorphism

$$
F G \longrightarrow F_{h} ; \quad \gamma \mapsto \gamma \cdot b
$$

of $F G$-modules restricts to an isomorphism $\varphi: \mathcal{O}_{F} G \cdot c \longrightarrow A_{h}$ of $\mathcal{O}_{F} G$ modules. Since $b$ is chosen to be self-dual, the map $\varphi$ is in fact a $G$-isometry over $\mathcal{O}_{F}$. Moreover, the lattice $\mathcal{O}_{F} G \cdot c$ is self-dual with respect to $t_{F}$ because $A_{h}$ is self-dual with respect to $\operatorname{Tr}_{h}$. It then follows from Proposition 3.5 (a) that $c \in J\left(F G_{(s)}\right)$, and from Proposition 3.10 (a) that $\left(A_{h}, \operatorname{Tr}_{h}\right) \in g\left(\mathcal{O}_{F} G\right)_{s}$. In particular, we have $\operatorname{ucl}\left(A_{h}\right)=\operatorname{ucl}\left(\left(\mathcal{O}_{F} G \cdot c, t_{F}\right)\right)=j_{(s)}(c)$. Recall also that the resolvend $\operatorname{map} \mathbf{r}_{G}: \operatorname{Map}\left(G, F_{v}^{c}\right) \longrightarrow F_{v}^{c} G$ is an isomorphism of $F_{v}^{c} G$ modules for each $v \in M_{F}$. Thus, equation (5.3) is equivalent to

$$
\mathbf{r}_{G}\left(a_{v}\right)=c_{v} \cdot \mathbf{r}_{G}(b)
$$

With this observation, we are now ready to prove Theorem 1.1.

\subsection{Proof of Theorem 1.1.}

Proof of Theorem 1.1. To prove (a), let $h \in H_{w}^{1}\left(\Omega_{K}, G\right)$. The fact that $h^{-1} \in$ $H_{w}^{1}\left(\Omega_{K}, G\right)$ is a direct consequence of [8, Proposition 5.1 (a)].

Let $b \in K_{h}$ be as in (5.2), where we choose $b$ to be self-dual. Moreover, for each $v \in M_{K}$, let $a_{v} \in A_{h_{v}}$ and $c_{v} \in\left(K_{v} G\right)^{\times}$be as in (5.1) and (5.3), respectively. We have $c:=\left(c_{v}\right) \in J\left(K G_{(s)}\right)$ and $\operatorname{ucl}\left(A_{h}\right)=j_{(s)}(c)$, as explained in Subsection 5.1.

Now, note that $\mathbf{r}_{G}(b) \in\left(K^{c} G\right)^{\times}$by Proposition 4.7 (a). Because $\mathbf{r}_{G}$ is bijective, there exists $b^{\prime} \in \operatorname{Map}\left(G, K^{c}\right)$ such that

$$
\mathbf{r}_{G}\left(b^{\prime}\right)=\mathbf{r}_{G}(b)^{-1} .
$$


By (4.3) and Proposition 4.7 (a), in fact $b^{\prime} \in K_{h^{-1}}$ and $K_{h^{-1}}=K G \cdot b^{\prime}$. Moreover, by Proposition 4.8 (b), clearly $b^{\prime}$ is self-dual with respect to $T r_{h^{-1}}$. Next, for each $v \in M_{K}$, there exists $a_{v}^{\prime} \in A_{h_{v}^{-1}}$ such that $A_{h_{v}^{-1}}=\mathcal{O}_{K_{v}} G \cdot a_{v}^{\prime}$ and

$$
\mathbf{r}_{G}\left(a_{v}^{\prime}\right)=\mathbf{r}_{G}\left(a_{v}\right)^{-1}
$$

by [8, Proposition 5.1 (b)]. Then, for each $v \in M_{K}$, equation (5.4) implies that

$$
\mathbf{r}_{G}\left(a_{v}^{\prime}\right)=c_{v}^{-1} \cdot \mathbf{r}_{G}\left(b^{\prime}\right)
$$

and so $a_{v}^{\prime}=c_{v}^{-1} \cdot b^{\prime}$. As in Subsection 5.1, this implies that $\operatorname{ucl}\left(A_{h^{-1}}\right)=$ $j_{(s)}\left(c^{-1}\right)=\operatorname{ucl}\left(A_{h}\right)^{-1}$, which proves $(\mathrm{a})$.

Next, to prove (b), let $h_{1}, h_{2} \in H_{w}^{1}\left(\Omega_{K}, G\right)$ be such that $d\left(h_{1}\right) \cap d\left(h_{2}\right)=\emptyset$. The fact that $h_{1} h_{2} \in H_{w}^{1}\left(\Omega_{K}, G\right)$ is a direct consequence of [8, Proposition $5.3(\mathrm{~b})]$.

For $i \in\{1,2\}$, let $b_{i} \in K_{h_{i}}$ be as in (5.2), where we choose $b_{i}$ to be selfdual. Moreover, for each $v \in M_{K}$, let $a_{i, v} \in A_{\left(h_{i}\right)_{v}}$ and $c_{i, v} \in\left(K_{v} G\right)^{\times}$be as in (5.1) and (5.3), respectively. As explained in Subsection 5.1, we have $c_{i}:=\left(c_{i, v}\right) \in J\left(K G_{(s)}\right)$ and $j_{(s)}\left(c_{i}\right)=\operatorname{ucl}\left(A_{h_{i}}\right)$.

Now, because $\mathbf{r}_{G}$ is bijective, there exists $b \in \operatorname{Map}\left(G, K^{c}\right)$ such that

$$
\mathbf{r}_{G}(b)=\mathbf{r}_{G}\left(b_{1}\right) \mathbf{r}_{G}\left(b_{2}\right) .
$$

By (4.3) and Proposition 4.7 (a), in fact $b \in K_{h_{1} h_{2}}$ and $K_{h_{1} h_{2}}=K G \cdot b$. Moreover, by Proposition 4.8 (b), clearly $b$ is self-dual with respect to $T r_{h_{1} h_{2}}$. Observe that for each $v \in M_{K}$, either $\left(h_{1}\right)_{v}$ or $\left(h_{2}\right)_{v}$ is unramified because $d\left(h_{1}\right) \cap d\left(h_{2}\right)=\emptyset$. So, there exists $a_{v} \in A_{\left(h_{1} h_{2}\right)_{v}}$ such that $A_{\left(h_{1} h_{2}\right)_{v}}=\mathcal{O}_{K_{v}} G \cdot a_{v}$ and

$$
\mathbf{r}_{G}\left(a_{v}\right)=\mathbf{r}_{G}\left(a_{1, v}\right) \mathbf{r}_{G}\left(a_{2, v}\right)
$$

by $\left[8\right.$, Proposition 5.3 (c)]. Then, for each $v \in M_{K}$, equation (5.4) implies that

$$
\mathbf{r}_{G}\left(a_{v}\right)=c_{1, v} c_{2, v} \cdot \mathbf{r}_{G}(b)
$$

and so $a_{v}=c_{1, v} c_{2, v} \cdot b$. As in Subsection 5.1, this implies that $\operatorname{ucl}\left(A_{h_{1} h_{2}}\right)=$ $j_{(s)}\left(c_{1} c_{2}\right)=\operatorname{ucl}\left(A_{h_{1}}\right) \operatorname{ucl}\left(A_{h_{2}}\right)$, which proves $(\mathrm{b})$. 
5.3. Summary of Main Ideas. Let $F$ be a number field. Moreover, assume that $G$ is abelian and of odd order. To prove Theorems 1.2 and 1.3, we will compute and characterize the $A$-realizable classes in $\operatorname{UCl}\left(\mathcal{O}_{F} G\right)$. Since most of the work has already been done in [8], below we will only sketch the main ideas involved.

Let $h \in \operatorname{Hom}\left(\Omega_{F}, G\right)$ be weakly ramified. As in Subsection 5.1, let $b \in F_{h}$ be a self-dual element such that $F_{h}=F G \cdot b$. Moreover, for each $v \in M_{F}$, let $a_{v} \in A_{h_{v}}$ be such that $A_{h_{v}}=\mathcal{O}_{F_{v}} G \cdot a_{v}$ and let $c_{v} \in\left(F_{v} G\right)^{\times}$be such that $a_{v}=c_{v} \cdot b$. Then, we have $c:=\left(c_{v}\right) \in J\left(F G_{(s)}\right)$ and $j_{(s)}(c)=\operatorname{ucl}\left(A_{h}\right)$. Recall also from (5.4) that the equation $a_{v}=c_{v} \cdot b$ is equivalent to

$$
\mathbf{r}_{G}\left(a_{v}\right)=c_{v} \cdot \mathbf{r}_{G}(b) .
$$

The resolvend $\mathbf{r}_{G}(b)$ of a self-dual element $b \in F_{h}$ satisfying $F_{h}=F G \cdot b$ is already characterized by Propositions 4.7 (a) and 4.8 (b). Hence, in order to characterize the class ucl $\left(A_{h}\right)$, it suffices to characterize the resolvend $\mathbf{r}_{G}\left(a_{v}\right)$ of an element $a_{v} \in A_{h_{v}}$ satisfying $A_{h_{v}}=\mathcal{O}_{F_{v}} G \cdot a_{v}$ for each $v \in M_{F}$. In fact, we will use reduced resolvends, which we define in Subsection 5.4.

For each $v \in M_{F}$, the resolvend $\mathbf{r}_{G}\left(a_{v}\right)$ of an element $a_{v} \in A_{h_{v}}$ satisfying $A_{h_{v}}=\mathcal{O}_{F_{v}} G \cdot a_{v}$ may be computed as follows. First of all, by [8, Proposition $9.2]$, we may write $h_{v}=h_{v, 1} h_{v, 2}$ for some $h_{v, 1}, h_{v, 2} \in \operatorname{Hom}\left(\Omega_{F_{v}}, G\right)$ such that $h_{v, 1}$ is unramified and $F_{v}^{h_{v, 2}} / F_{v}$ is totally ramified. Then, using [8, Proposition 5.3 (c)], we may decompose

$$
\mathbf{r}_{G}\left(a_{v}\right)=\mathbf{r}_{G}\left(a_{v, 1}\right) \mathbf{r}_{G}\left(a_{v, 2}\right)
$$

where $\mathcal{O}_{h_{v, 1}}=\mathcal{O}_{F_{v}} G \cdot a_{v, 1}$ and $A_{h_{v, 2}}=\mathcal{O}_{F_{v}} G \cdot a_{v, 2}$. The resolvend $\mathbf{r}_{G}\left(a_{v, 1}\right)$ of such an element $a_{v, 1}$ is already characterized by Proposition 4.7 (b). On the other hand, the resolvend $\mathbf{r}_{G}\left(a_{v, 2}\right)$ may be described using the modified Stickelberger transpose (see [8, Propositions 10.2 and 13.2]), which we define in Subsection 5.5. Using results already proved in [8], we will give a complete characterization of the set

$$
\mathcal{A}_{u}^{t}\left(\mathcal{O}_{F} G\right):=\left\{\operatorname{ucl}\left(A_{h}\right): h \in H_{t}^{1}\left(\Omega_{F}, G\right)\right\}
$$

of tame $A$-realizable classes in $\operatorname{UCl}\left(\mathcal{O}_{F} G\right)$ in (5.17). 
5.4. Cohomology and Reduced Resolvends. Let $F$ be a number field or a finite extension of $\mathbb{Q}_{p}$. Moreover, assume that $G$ is abelian.

First of all, following [5, Sections 1 and 2], we will use cohomology to define reduced resolvends. Recall that $\Omega_{F}$ acts trivially on $G$ and define

$$
\mathcal{H}(F G):=\left(\left(F^{c} G\right)^{\times} / G\right)^{\Omega_{F}} .
$$

Taking $\Omega_{F}$-cohomology of the exact sequence

$$
1 \longrightarrow G \longrightarrow\left(F^{c} G\right)^{\times} \longrightarrow\left(F^{c} G\right)^{\times} / G \longrightarrow 1
$$

yields the exact sequence

$$
1 \longrightarrow G \longrightarrow(F G)^{\times} \longrightarrow \mathcal{H}(F G) \stackrel{\delta}{\longrightarrow} \operatorname{Hom}\left(\Omega_{F}, G\right) \longrightarrow 1
$$

where exactness on the right follows from the fact that $H^{1}\left(\Omega_{F},\left(F^{c} G\right)^{\times}\right)=1$, which is Hilbert's Theorem 90. Alternatively, notice that a coset $\mathbf{r}_{G}(a) G \in$ $\mathcal{H}(F G)$ lies in the preimage of $h \in \operatorname{Hom}\left(\Omega_{F}, G\right)$ if and only if

$$
h(\omega)=\mathbf{r}_{G}(a)^{-1}\left(\omega \cdot \mathbf{r}_{G}(a)\right) \quad \text { for all } \omega \in \Omega_{F},
$$

which is equivalent to $F_{h}=F G \cdot a$ by (4.4) and Proposition 4.7 (a). By the Normal Basis Theorem, for any $h \in \operatorname{Hom}\left(\Omega_{F}, G\right)$ there always exists $a \in F_{h}$ for which $F_{h}=F G \cdot a$. This shows that $\delta$ is indeed surjective.

The same argument as above also shows that

$$
\mathcal{H}(F G)=\left\{r_{G}(a) \mid F_{h}=F G \cdot a \text { for some } h \in \operatorname{Hom}\left(\Omega_{F}, G\right)\right\}
$$

Similarly, we may define

$$
\mathcal{H}\left(\mathcal{O}_{F} G\right):=\left(\left(\mathcal{O}_{F^{c}} G\right)^{\times} / G\right)^{\Omega_{F}} .
$$

Then, the argument above together with Proposition 4.7 (b) imply that

$$
\mathcal{H}\left(\mathcal{O}_{F} G\right)=\left\{r_{G}(a) \mid \mathcal{O}_{h}=\mathcal{O}_{F} G \cdot a \text { for some } h \in \operatorname{Hom}\left(\Omega_{F}, G\right) \text { unramified }\right\} .
$$

In view of Proposition 4.8, we will further define

$$
\begin{aligned}
& \mathcal{H}\left(F G_{(s)}\right):=\left\{r_{G}(a) \in \mathcal{H}(F G) \mid \mathbf{r}_{G}(a) \mathbf{r}_{G}(a)^{[-1]} \in\left(\mathcal{O}_{F} G\right)^{\times}\right\} ; \\
& \mathcal{H}\left(F G_{(1)}\right):=\left\{r_{G}(a) \in \mathcal{H}(F G) \mid \mathbf{r}_{G}(a) \mathbf{r}_{G}(a)^{[-1]}=1\right\},
\end{aligned}
$$


which are clearly subgroups of $\mathcal{H}(F G)$. Moreover, it is clear that both of the conditions $\mathbf{r}_{G}(a) \mathbf{r}_{G}(a)^{[-1]} \in\left(\mathcal{O}_{F} G\right)^{\times}$and $\mathbf{r}_{G}(a) \mathbf{r}_{G}(a)^{[-1]}=1$ are independent of the choice of the representative $\mathbf{r}_{G}(a)$.

Definition 5.1. Let $\mathbf{r}_{G}(a) G \in \mathcal{H}(F G)$. Define

$$
r_{G}(a):=\mathbf{r}_{G}(a) G
$$

called the reduced resolvend of $a$. Moreover, define $h_{a} \in \operatorname{Hom}\left(\Omega_{F}, G\right)$ by

$$
h_{a}(\omega):=\mathbf{r}_{G}(a)^{-1}\left(\omega \cdot \mathbf{r}_{G}(a)\right),
$$

called the homomorphism associated to $r_{G}(a)$. This definition is independent of the choice of the representative $\mathbf{r}_{G}(a)$, and we have $F_{h}=F G \cdot a$ by Proposition 4.7 (a) and (4.4).

Definition 5.2. For $F$ a number field, let $J(\mathcal{H}(F G))$ and $J\left(\mathcal{H}\left(F G_{(s)}\right)\right)$ be the restricted direct products of the groups $\mathcal{H}\left(F_{v} G\right)$ and $\mathcal{H}\left(F_{v} G_{(s)}\right)$, respectively, with respect to the subgroups $\mathcal{H}\left(\mathcal{O}_{F_{v}} G\right)$ for $v \in M_{F}$. Moreover, let

$$
\eta: \mathcal{H}(F G) \longrightarrow J(\mathcal{H}(F G))
$$

be the diagonal map and let

$$
U\left(\mathcal{H}\left(\mathcal{O}_{F} G\right)\right):=\prod_{v \in M_{F}} \mathcal{H}\left(\mathcal{O}_{F_{v}} G\right)
$$

be the group of unit idèles.

Next, we explain how reduced resolvends may be interpreted as functions on characters of $G$. To that end, define $\operatorname{det}: \mathbb{Z} \widehat{G} \longrightarrow \widehat{G}$ by

$$
\operatorname{det}\left(\sum_{\chi} n_{\chi} \chi\right):=\prod_{\chi} \chi^{n_{\chi}}
$$

and set

$$
S_{\widehat{G}}:=\operatorname{ker}(\operatorname{det})
$$

Then, applying the functor $\operatorname{Hom}\left(-,\left(F^{c}\right)^{\times}\right)$to the short exact sequence

$$
1 \longrightarrow S_{\widehat{G}} \longrightarrow \mathbb{Z} \widehat{G} \stackrel{\operatorname{det}}{\longrightarrow} \widehat{G} \longrightarrow 1
$$


yields the short exact sequence

$$
1 \rightarrow \operatorname{Hom}\left(\widehat{G},\left(F^{c}\right)^{\times}\right) \rightarrow \operatorname{Hom}\left(\mathbb{Z} \widehat{G},\left(F^{c}\right)^{\times}\right) \rightarrow \operatorname{Hom}\left(S_{\widehat{G}},\left(F^{c}\right)^{\times}\right) \rightarrow 1,
$$

where exactness on the right follows from the fact that $\left(F^{c}\right)^{\times}$is divisible and thus injective.

Observe that we have canonical identifications

$$
\left(F^{c} G\right)^{\times}=\operatorname{Map}\left(\widehat{G},\left(F^{c}\right)^{\times}\right)=\operatorname{Hom}\left(\mathbb{Z} \widehat{G},\left(F^{c}\right)^{\times}\right) .
$$

The second identification is given by extending the maps $\widehat{G} \longrightarrow\left(F^{c}\right)^{\times}$via $\mathbb{Z}$-linearity, and the first is induced by characters (see [8, (7.7) and (7.8)], for example). Since $G=\operatorname{Hom}\left(\widehat{G},\left(F^{c}\right)^{\times}\right)$canonically, the thirds terms

$$
\left(F^{c} G\right)^{\times} / G=\operatorname{Hom}\left(S_{\widehat{G}},\left(F^{c}\right)^{\times}\right)
$$

in (5.7) and (5.9), respectively, are naturally identified as well. Taking $\Omega_{F^{-}}$ invariants, we then obtain the identification

$$
\mathcal{H}(F G)=\operatorname{Hom}_{\Omega_{F}}\left(S_{\widehat{G}},\left(F^{c}\right)^{\times}\right) .
$$

Under this identification, we have

$$
\mathcal{H}\left(\mathcal{O}_{F} G\right) \subset \operatorname{Hom}_{\Omega_{F}}\left(S_{\widehat{G}}, \mathcal{O}_{F^{c}}^{\times}\right)
$$

This inclusion is an equality when $F$ is a finite extension of $\mathbb{Q}_{p}$, where $p$ does not divide $|G|$ (see [8, Proposition 7.4], for example).

Finally, we will define

$$
\operatorname{rag}_{F}:(F G)^{\times} \longrightarrow \mathcal{H}(F G)
$$

to be the homomorphism induced by the quotient map $(F G)^{\times} \longrightarrow(F G)^{\times} / G$.

Definition 5.3. For $F$ a number field, observe that the homomorphism

$$
\prod_{v \in M_{F}} \operatorname{rag}_{F_{v}}: J(F G) \longrightarrow J(\mathcal{H}(F G))
$$


is clearly well-defined, and that the diagram

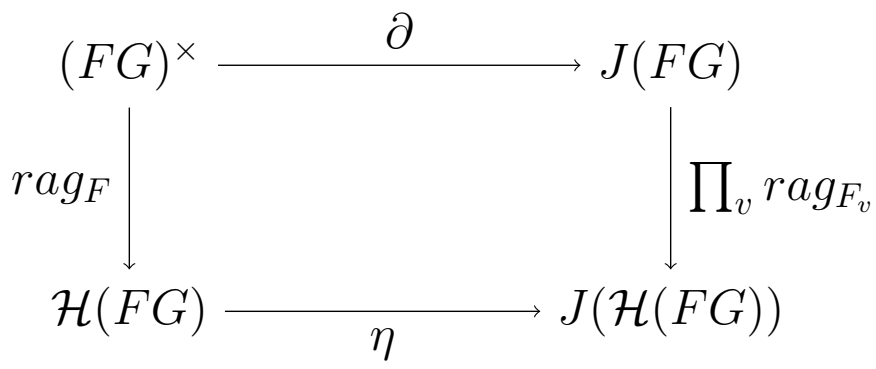

commutes. By abuse of notation, we will denote the map in (5.12) by $r a g=$ $\operatorname{rag}_{F}$.

5.5. The Modified Stickelberger Transpose. Let $F$ be a number field or a finite extension of $\mathbb{Q}_{p}$. Moreover, assume that $G$ is abelian and of odd order.

We will recall the definition of the modified Stickelberger transpose, which was introduced by the author in [8, Section 8]. Recall that we chose a compatible set $\left\{\zeta_{n}: n \in \mathbb{Z}^{+}\right\}$of primitive roots of unity in $F^{c}$.

Definition 5.4. For each $\chi \in \widehat{G}$ and $s \in G$, let

$$
v(\chi, s) \in\left[\frac{1-|s|}{2}, \frac{|s|-1}{2}\right]
$$

be the unique integer (recall that $G$ has odd order) such that

$$
\chi(s)=\left(\zeta_{|s|}\right)^{v(\chi, s)}
$$

and define

$$
\langle\chi, s\rangle_{*}:=v(\chi, s) /|s| \text {. }
$$

Extending this definition by $\mathbb{Q}$-linearity, we obtain a pairing

$$
\langle,\rangle_{*}: \mathbb{Q} \widehat{G} \times \mathbb{Q} G \longrightarrow \mathbb{Q},
$$

called the modified Stickelberger pairing. The map

$$
\Theta_{*}: \mathbb{Q} \widehat{G} \longrightarrow \mathbb{Q} G ; \quad \Theta_{*}(\psi):=\sum_{s \in G}\langle\psi, s\rangle_{*} s
$$

is called the modified Stickelberger map.

Proposition 5.5. For $\psi \in \mathbb{Z} \widehat{G}$, we have $\Theta_{*}(\psi) \in \mathbb{Z} G$ if and only if $\psi \in S_{\widehat{G}}$. Proof. See [8, Proposition 8.2]. 
Up until now, we have let $\Omega_{F}$ act trivially on $G$. Below, we introduce other $\Omega_{F}$-actions on $G$, one of which will make the $\mathbb{Q}$-linear map $\Theta_{*}: \mathbb{Q} \widehat{G} \longrightarrow \mathbb{Q} G$ preserve $\Omega_{F}$-action. Here, the $\Omega_{F}$-action on $\widehat{G}$ is the canonical one induced by the $\Omega_{F}$-action on the roots of unity.

Definition 5.6. Let $m=\exp (G)$ and let $\mu_{m}$ be the group of $m$-th roots of unity in $F^{c}$. The $m$-th cyclotomic character of $\Omega_{F}$ is the homomorphism

$$
\kappa: \Omega_{F} \longrightarrow(\mathbb{Z} / m \mathbb{Z})^{\times}
$$

defined by the equations

$$
\omega(\zeta)=\zeta^{\kappa(\omega)} \quad \text { for } \omega \in \Omega_{F} \text { and } \zeta \in \mu_{m} .
$$

For $n \in \mathbb{Z}$, let $G(n)$ be the group $G$ equipped with the $\Omega_{F}$-action given by

$$
\omega \cdot s:=s^{\kappa\left(\omega^{n}\right)} \quad \text { for } s \in G \text { and } \omega \in \Omega_{F} .
$$

We will need $G(-1)$. But of course, if $F$ contains the $m$-th roots of unity, then $\kappa$ is trivial and $G(n)=G(0)$ is equipped with the trivial $\Omega_{F}$-action for all $n \in \mathbb{Z}$.

Proposition 5.7. The map $\Theta_{*}: \mathbb{Q} \widehat{G} \longrightarrow \mathbb{Q} G(-1)$ preserves $\Omega_{F}$-action.

Proof. See [8, Proposition 8.4].

From Propositions 5.5 and 5.7, we obtain an $\Omega_{F^{-} \text {equivariant map }}$

$$
\Theta_{*}: S_{\widehat{G}} \longrightarrow \mathbb{Z} G(-1)
$$

Applying the functor $\operatorname{Hom}\left(-,\left(F^{c}\right)^{\times}\right)$then yields an $\Omega_{F^{-} \text {equivariant homo- }}$ morphism

$$
\Theta_{*}^{t}: \operatorname{Hom}\left(\mathbb{Z} G(-1),\left(F^{c}\right)^{\times}\right) \longrightarrow \operatorname{Hom}\left(S_{\widehat{G}},\left(F^{c}\right)^{\times}\right) ; \quad f \mapsto f \circ \Theta_{*} .
$$

Via restriction, we then obtain a homomorphism

$$
\Theta_{*}^{t}=\Theta_{*, F}^{t}: \operatorname{Hom}_{\Omega_{F}}\left(\mathbb{Z} G(-1),\left(F^{c}\right)^{\times}\right) \longrightarrow \operatorname{Hom}_{\Omega_{F}}\left(S_{\widehat{G}},\left(F^{c}\right)^{\times}\right),
$$

called the modified Stickelberger transpose. 
Notice that we have a natural identification

$$
\operatorname{Hom}_{\Omega_{F}}\left(\mathbb{Z} G(-1),\left(F^{c}\right)^{\times}\right)=\operatorname{Map}_{\Omega_{F}}\left(G(-1),\left(F^{c}\right)^{\times}\right) .
$$

To simplify notation, let

$$
\begin{aligned}
\Lambda(F G) & :=\operatorname{Map}_{\Omega_{F}}\left(G(-1), F^{c}\right) \\
\Lambda\left(\mathcal{O}_{F} G\right) & :=\operatorname{Map}_{\Omega_{F}}\left(G(-1), \mathcal{O}_{F^{c}}\right) .
\end{aligned}
$$

Then, we may view $\Theta_{*}^{t}$ as a homomorphism

$$
\Theta_{*}^{t}: \Lambda(F G)^{\times} \longrightarrow \mathcal{H}(F G)
$$

(recall the identification in (5.10)).

Proposition 5.8. We have $\Theta_{*}^{t}\left(\Lambda(F G)^{\times}\right) \subset \mathcal{H}\left(F G_{(1)}\right)$.

Proof. See [8, Proposition 8.5].

Definition 5.9. For $F$ a number field, let $J(\Lambda(F G))$ be the restricted direct product of the groups $\Lambda\left(F_{v} G\right)^{\times}$with respect to the subgroups $\Lambda\left(\mathcal{O}_{F_{v}} G\right)^{\times}$for $v \in M_{F}$. Moreover, let

$$
\lambda: \Lambda(F G)^{\times} \longrightarrow J(\Lambda(F G))
$$

be the diagonal map and let

$$
U\left(\Lambda\left(\mathcal{O}_{F} G\right)\right):=\prod_{v \in M_{F}} \Lambda\left(\mathcal{O}_{F_{v}} G\right)^{\times}
$$

be the group of unit idèles.

Next, observe that the homomorphism

$$
\prod_{v \in M_{F}} \Theta_{*, F_{v}}^{t}: J(\Lambda(F G)) \longrightarrow J(\mathcal{H}(F G))
$$

is well-defined since the inclusion (5.11) is an equality for all but finitely many $v \in M_{F}$. Because we chose $\left\{i_{v}\left(\zeta_{n}\right): n \in \mathbb{Z}^{+}\right\}$to be the compatible set of 
primitive roots of unity in $F_{v}^{c}$, the diagram

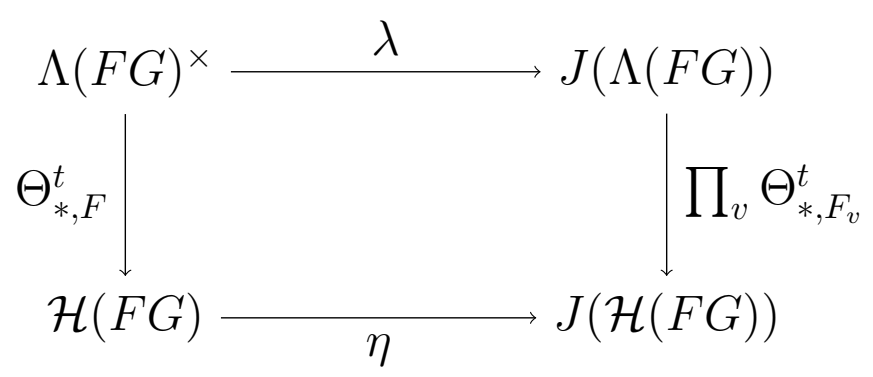

commutes. By abuse of notation, we will denote the map in (5.13) by $\Theta_{*}^{t}=$ $\Theta_{*, F}^{t}$.

5.6. Approximation Theorems. Let $F$ be a number field. Moreover, assume that $G$ is abelian and of odd order.

First, we will give a preliminary characterization of the set $\mathcal{A}_{u}^{t}\left(\mathcal{O}_{F} G\right)$ using results already proved in [8]. To that end, we need one further definition (see $[8$, Section 10] for the motivation of the definition).

Definition 5.10. For each $v \in M_{F}$, choose a uniformizer $\pi_{F_{v}}$ in $F_{v}$ and let $q_{F_{v}}$ be the order of the residue field $\mathcal{O}_{F_{v}} /\left(\pi_{F_{v}}\right)$. For each $s \in G$ of order dividing $q_{F_{v}}-1$, define

$$
f_{F_{v}, s} \in \Lambda\left(F_{v} G\right)^{\times} ; \quad f_{F_{v}, s}(t):= \begin{cases}\pi_{F_{v}} & \text { if } t=s \neq 1 \\ 1 & \text { otherwise. }\end{cases}
$$

Notice that $f_{F_{v}, s}$ indeed preserves $\Omega_{F_{v}}$-action because all $\left(q_{F_{v}}-1\right)$-st roots of unity are contained in $F_{v}$, whence elements in $G$ of order dividing $q_{F_{v}}-1$ are fixed by $\Omega_{F_{v}}$, as is $\pi_{F_{v}}$. Let $\mathfrak{F}_{F_{v}}$ be the set of all such $f_{F_{v}, s}$ and define

$$
\mathfrak{F}=\mathfrak{F}_{F}:=\left\{f \in J(\Lambda(F G)) \mid f_{v} \in \mathfrak{F}_{F_{v}} \text { for all } v \in M_{F}\right\} .
$$

Analogous to [8, Theorem 11.2], we have the following theorem.

Theorem 5.11. Let $h \in H o m\left(\Omega_{F}, G\right)$ and $F_{h}=F G \cdot b$ with $b$ self-dual. Then, we have $h$ is tame if and only if there exists $c \in J\left(F G_{(s)}\right)$ such that

$$
\operatorname{rag}(c)=\eta\left(r_{G}(b)\right)^{-1} u \Theta_{*}^{t}(f)
$$

for some $u \in U\left(\mathcal{H}\left(\mathcal{O}_{F} G\right)\right)$ and $f \in \mathfrak{F}$. Moreover, if (5.15) holds, then 
(1) $s_{v} \in h_{v}\left(\Omega_{F_{v}}\right)$, where $f_{v}=f_{F_{v}, s_{v}}$, for all $v \in M_{F}$;

(2) $f_{v}=1$ if and only if $h_{v}$ is unramified for all $v \in M_{F}$;

(3) $j_{(s)}(c)=u c l\left(A_{h}\right)$.

Proof. First, assume that $h$ is tame. Then, by [8, Theorem 11.2], there exists $c \in J(F G)$ such that (5.15) holds for some $u \in U\left(\mathcal{H}\left(\mathcal{O}_{F} G\right)\right)$ and $f \in \mathfrak{F}$. It is clear that $U\left(\mathcal{H}\left(\mathcal{O}_{F} G\right)\right) \subset J\left(\mathcal{H}\left(F G_{(s)}\right)\right.$, and we have $\Theta_{*}^{t}(\mathfrak{F}) \subset J\left(\mathcal{H}\left(F G_{(s)}\right)\right.$ by Proposition 5.8. Since $b$ is self-dual, it follows from Proposition 4.8 that in fact $c \in J\left(F G_{(s)}\right)$, which proves the claim.

Conversely, assume that there exists $c \in J\left(F G_{(s)}\right)$ such that (5.15) holds for some $u \in U\left(\mathcal{H}\left(\mathcal{O}_{F} G\right)\right)$ and $f \in \mathfrak{F}$. By [8, Theorem 11.2], this implies that $h$ is tame, and that (1) and (2) hold. To show that (3) holds as well, notice that for each $v \in M_{F}$, there exists $a_{v} \in A_{h_{v}}$ such that $A_{h_{v}}=\mathcal{O}_{F_{v}} G \cdot a_{v}$ and

$$
r_{G}\left(a_{v}\right)=u_{v} \Theta_{*}^{t}\left(f_{v}\right)
$$

by [8, Theorem 10.4]. In particular, we have $r_{G}\left(a_{v}\right)=\operatorname{rag}\left(c_{v}\right) r_{G}(b)$, so there exists $t_{v} \in G$ such that

$$
\mathbf{r}_{G}\left(a_{v}\right)=c_{v} \mathbf{r}_{G}(b) t_{v}=c_{v} t_{v} \cdot \mathbf{r}_{G}(b) .
$$

This implies that $a_{v}=\left(c_{v} t_{v}\right) \cdot b$. Now, set $t:=\left(t_{v}\right) \in U\left(\mathcal{O}_{F} G\right)$. Since $b$ is self-dual, as in Subsection 5.1, we deduce that $\operatorname{ucl}\left(A_{h}\right)=j_{(s)}(c t)=j_{(s)}(c)$. This proves (3) and completes the proof of the theorem.

Remark 5.12. The decomposition of $\operatorname{rag}(c)$ given by (5.15) in Theorem 5.11 comes from equation (5.5) and decomposition (5.6) in Subsection 5.3.

Theorem 5.11 implies that for $c \in J\left(F G_{(s)}\right)$, we have $j_{(s)}(c) \in \mathcal{A}_{u}^{t}\left(\mathcal{O}_{F} G\right)$ if and only if $\operatorname{rag}(c)$ is an element of

$$
\eta\left(\mathcal{H}\left(F G_{(1)}\right)\right) U\left(\mathcal{H}\left(\mathcal{O}_{F} G\right)\right) \Theta_{*}^{t}(\mathfrak{F})
$$

(recall Proposition 3.5 (b)). However, it is unclear whether (5.16) is a subgroup of $J(\mathcal{H}(F G))$ because $\mathfrak{F}$ is only a subset of $J(\Lambda(F G))$. Below, we state two approximation theorems. They will allow us to replace $\mathfrak{F}$ by $J(\Lambda(F G))$ in (5.16), which will in turn allow us to prove Theorems 1.2 and 1.3.

First, we need some further definitions. 
Definition 5.13. Let $\mathfrak{m}$ be an ideal in $\mathcal{O}_{F}$. For each $v \in M_{F}$, let

$$
\begin{aligned}
U_{\mathfrak{m}}\left(\mathcal{O}_{F_{v}^{c}}\right) & :=\left(1+\mathfrak{m} \mathcal{O}_{F_{v}^{c}}\right) \cap\left(\mathcal{O}_{F_{v}^{c}}\right)^{\times} ; \\
U_{\mathfrak{m}}^{\prime}\left(\Lambda\left(\mathcal{O}_{F_{v}} G\right)\right) & :=\left\{g_{v} \in \Lambda\left(\mathcal{O}_{F_{v}} G\right)^{\times} \mid g_{v}(s) \in U_{\mathfrak{m}}\left(\mathcal{O}_{F_{v}^{c}}\right) \text { for all } s \in G \text { with } s \neq 1\right\},
\end{aligned}
$$

Moreover, set

$$
U_{\mathfrak{m}}^{\prime}\left(\Lambda\left(\mathcal{O}_{F} G\right)\right):=\left(\prod_{v \in M_{F}} U_{\mathfrak{m}}^{\prime}\left(\Lambda\left(\mathcal{O}_{F_{v}} G\right)\right)\right) \cap J(\Lambda(F G)) .
$$

The modified ray class group $\bmod \mathfrak{m}$ of $\Lambda(F G)$ is defined by

$$
\mathrm{Cl}_{\mathfrak{m}}^{\prime}(\Lambda(F G)):=\frac{J(\Lambda(F G))}{\lambda\left(\Lambda(F G)^{\times}\right) U_{\mathfrak{m}}^{\prime}\left(\Lambda\left(\mathcal{O}_{F} G\right)\right)} .
$$

Definition 5.14. For $g \in J(\Lambda(F G))$ and $s \in G$, define

$$
g_{s}:=\prod_{v \in M_{F}} g_{v}(s) \in \prod_{v \in M_{F}}\left(F_{v}^{c}\right)^{\times} .
$$

We can now state the approximation theorems.

Theorem 5.15. Let $\mathfrak{m}$ be an ideal in $\mathcal{O}_{F}$ divisible by both $|G|$ and $\exp (G)^{2}$.

Then, we have

$$
\Theta_{*}^{t}\left(U_{\mathfrak{m}}^{\prime}\left(\Lambda\left(\mathcal{O}_{F} G\right)\right) \subset U\left(\mathcal{H}\left(\mathcal{O}_{F} G\right)\right)\right.
$$

Proof. See [8, Theorem 11.5 (b)].

Theorem 5.16. Let $g \in J(\Lambda(F G))$ and let $T$ be a finite subset of $M_{F}$. Then, there exists $f \in \mathfrak{F}$ such that $f_{v}=1$ for all $v \in T$ and

$$
g \equiv f \quad\left(\bmod \lambda\left(\Lambda(F G)^{\times}\right) U_{\mathfrak{m}}^{\prime}\left(\Lambda\left(\mathcal{O}_{F} G\right)\right)\right) .
$$

Moreover, we may choose $f$ so that for each $s \in G(-1)$ with $s \neq 1$, there exists $\omega \in \Omega_{F}$ such that $f_{\omega \cdot s} \neq 1$.

Proof. See [5, Proposition 6.14].

\subsection{Proof of Theorems 1.2 and $\mathbf{1 . 3 .}$}

Proof of Theorem 1.2. Let $\rho_{u}$ be the composition of the homomorphism

$$
J\left(K G_{(s)}\right) \longrightarrow J\left(\mathcal{H}\left(K G_{(s)}\right)\right) ; \quad c \mapsto \operatorname{rag}(c),
$$


where rag is as in Definition 5.3, followed by the natural quotient map

$$
J\left(\mathcal{H}\left(K G_{(s)}\right)\right) \longrightarrow \frac{J\left(\mathcal{H}\left(K G_{(s)}\right)\right)}{\eta\left(\mathcal{H}\left(K G_{(1)}\right)\right) U\left(\mathcal{H}\left(\mathcal{O}_{K} G\right)\right) \Theta_{*}^{t}(J(\Lambda(K G)))} .
$$

Note that $\Theta_{*}^{t}(J(\Lambda(K G))) \subset J\left(\mathcal{H}\left(K G_{(s)}\right)\right)$ by Proposition 5.8. We will show that $\mathcal{A}_{u}^{t}\left(\mathcal{O}_{K} G\right)$ is a subgroup of $\operatorname{UCl}\left(\mathcal{O}_{K} G\right)$ by showing that

$$
j_{(s)}^{-1}\left(\mathcal{A}_{u}^{t}\left(\mathcal{O}_{K} G\right)\right)=\operatorname{ker}\left(\rho_{u}\right)
$$

or equivalently, that for $c \in J\left(K G_{(s)}\right)$, we have $j_{(s)}(c) \in \mathcal{A}_{u}^{t}\left(\mathcal{O}_{K} G\right)$ if and only if

$$
\operatorname{rag}(c) \in \eta\left(\mathcal{H}\left(K G_{(1)}\right)\right) U\left(\mathcal{H}\left(\mathcal{O}_{K} G\right)\right) \Theta_{*}^{t}(J(\Lambda(K G))) .
$$

To that end, let $c \in J\left(K G_{(s)}\right)$ be given. If $j_{(s)}(c)=\operatorname{ucl}\left(A_{h}\right)$ for some tame $h \in \operatorname{Hom}\left(\Omega_{K}, G\right)$, with $K_{h}=K G \cdot b$ and $b$ self-dual say, then $r_{G}(b) \in \mathcal{H}\left(K G_{(1)}\right)$ by (5.8) and Proposition 4.8 (b). Moreover, by Theorem 5.11, there exists $c^{\prime} \in J\left(K G_{(s)}\right)$ such that $j_{(s)}\left(c^{\prime}\right)=\operatorname{ucl}\left(A_{h}\right)$ and

$$
\operatorname{rag}\left(c^{\prime}\right) \in \eta\left(\mathcal{H}\left(K G_{(1)}\right)\right) U\left(\mathcal{H}\left(\mathcal{O}_{K} G\right)\right) \Theta_{*}^{t}(J(\Lambda(K G))) .
$$

Since $j_{(s)}(c)=\operatorname{ucl}\left(A_{h}\right)$ also, we have

$$
c \equiv c^{\prime} \quad\left(\bmod \partial\left(K G_{(1)}\right) U\left(\mathcal{O}_{K} G\right)\right) .
$$

It is then clear that (5.17) indeed holds.

Conversely, if (5.17) holds, then

$$
\operatorname{rag}(c)=\eta\left(r_{G}(b)\right)^{-1} u \Theta_{*}^{t}(g)
$$

for some $r_{G}(b) \in \mathcal{H}\left(K G_{(1)}\right), u \in U\left(\mathcal{H}\left(\mathcal{O}_{K} G\right)\right)$, and $g \in J(\Lambda(K G))$. Let $\mathfrak{m}$ be an ideal in $\mathcal{O}_{K}$. Then, by Theorem 5.16 , there exists $f \in \mathfrak{F}$ such that

$$
g \equiv f \quad\left(\bmod \lambda\left(\Lambda(K G)^{\times}\right) U_{\mathfrak{m}}^{\prime}\left(\Lambda\left(\mathcal{O}_{K} G\right)\right)\right) .
$$

Choosing $\mathfrak{m}$ to be divisible by $|G|$ and $\exp (G)^{2}$, by Proposition 5.8 and Theorem 5.15 , the above implies that

$$
\Theta_{*}^{t}(g) \equiv \Theta_{*}^{t}(f) \quad\left(\bmod \eta\left(\mathcal{H}\left(K G_{(1)}\right)\right) U\left(\mathcal{H}\left(\mathcal{O}_{K} G\right)\right)\right) .
$$


Hence, changing $b$ and $u$ in (5.18) if necessary, we may assume that $g=f$. Since $b$ is self-dual, if $h:=h_{b}$ is the homomorphism associated to $r_{G}(b)$, then $h$ is tame and $j_{(s)}(c)=\operatorname{ucl}\left(A_{h}\right)$ by Theorem 5.11. It remains to show that $h$ may be chosen such that (1) and (2) are satisfied.

Let $T$ be a finite set of primes in $\mathcal{O}_{K}$. First of all, by Theorem 5.16, we may choose the $f \in \mathfrak{F}$ in (5.19) such that $f_{v}=1$ for all $v \in T$. It then follows from Theorem 5.11 that $h_{v}$ is unramified for all $v \in T$, so (2) holds. Moreover, we may also choose the $f \in \mathfrak{F}$ in (5.19) such that for each $s \in G(-1)$ with $s \neq 1$, there exists $\omega \in \Omega_{K}$ such that $f_{\omega \cdot s} \neq 1$. In particular, we have $f_{v}=f_{K_{v}, \omega \cdot s}$ for some $v \in M_{K}$. But observe that $\langle s\rangle=\langle\omega \cdot s\rangle$ and that $\omega \cdot s \in h_{v}\left(\Omega_{K_{v}}\right)$ by Theorem 5.11, so $s \in h\left(\Omega_{K}\right)$. This shows that $h$ is surjective and hence $K_{h}$ is a field, so (1) holds as well. This completes the proof of the theorem.

Proof of Theorem 1.3. Let $h \in \operatorname{Hom}\left(\Omega_{K}, G\right)$ be given as in the statement of the theorem. Let $b \in K_{h}$ be as in (5.2), where we choose $b$ to be self-dual. Moreover, for each $v \in M_{K}$, let $a_{v} \in A_{h_{v}}$ and $c_{v} \in\left(K_{v} G\right)^{\times}$be given as in (5.1) and (5.3), respectively. Then, we have $c \in J\left(K G_{(s)}\right)$ and $\operatorname{ucl}\left(A_{h}\right)=j_{(s)}(c)$, as explained in Subsection 5.1. Moreover, equation (5.4) implies that

$$
\operatorname{rag}\left(c_{v}\right)=r_{G}(b)^{-1} r_{G}\left(a_{v}\right)
$$

for each $v \in M_{K}$. Notice also that $r_{G}(b) \in \mathcal{H}\left(K G_{(1)}\right)$ by (5.8) and Proposition 4.8 (b). From (5.17), we then see that $\operatorname{ucl}\left(A_{h}\right) \in \mathcal{A}_{u}^{t}\left(\mathcal{O}_{K} G\right)$ will hold provided that for all $v \in M_{K}$, we have

$$
r_{G}\left(a_{v}\right) \in \mathcal{H}\left(\mathcal{O}_{K_{v}} G\right) \Theta_{*}^{t}\left(\Lambda\left(K_{v} G\right)^{\times}\right)
$$

If $v \notin V$, then (5.20) follows from [8, Theorem 10.3]. If $v \in V$, then hypotheses (1) and (2) allow us to apply [8, Theorem 13.2] and conclude that (5.20) holds. This proves the theorem.

\section{ACKnowledgments}

I would like to thank my advisor Professor Adebisi Agboola for bringing this problem to my attention. 


\section{REFERENCES}

[1] E. Bayer-Fluckiger and H. W. Lenstra, Forms in odd degree extensions and self-dual normal bases, Amer. J. Math. 112(3) (1990) 359-373.

[2] B. Erez, The Galois structure of the square root of the inverse different, Math. Z. 208(2) (1991) 239-255.

[3] B. Erez and J. Morales, The Hermitian structure of rings of integers in odd degree abelian extension, J. Number Theory 41 (1992) 92-104.

[4] A. Fröhlich and M.J. Taylor, Algebraic number theory. Cambridge University Press, 1991.

[5] L.R. McCulloh, Galois module structure of abelian extensions, J. Reine. Angew. Math. 375/376 (1987) 259-306.

[6] J. Morales, Trace forms and Stickelberger relations, J. Number Theory 51 (1995) 118-129.

[7] J.P. Serre, Local fields, Graduate text in mathematics 6\%. Springer-Verlag, New York, 1979.

[8] C. Tsang, On the Galois module structure of the square root of the inverse different in abelian extensions, J. Number Theory 160 (2016), 759-804.

Department of Mathematics, University of California, Santa Barbara

E-mail address: cindytsy@math.ucsb.edu

$U R L:$ http://math.ucsb.edu/ cindytsy 